TRANSACTIONS OF THE

AMERICAN MATHEMATICAL SOCIETY

Volume 352, Number 4, Pages 1743-1757

S 0002-9947(99)02330-2

Article electronically published on July 1, 1999

\title{
A CONTINUOUS CIRCLE OF PSEUDO-ARCS FILLING UP THE ANNULUS
}

\author{
JANUSZ R. PRAJS
}

To the memory of Professor Bronistaw Knaster

\begin{abstract}
We prove an early announcement by Knaster on a decomposition of the plane. Then we establish an announcement by Anderson saying that the plane annulus admits a continuous decomposition into pseudo-arcs such that the quotient space is a simple closed curve. This provides a new plane curve, "a selectible circle of pseudo-arcs", and answers some questions of Lewis.
\end{abstract}

In 1922 the famous construction of an hereditarily indecomposable plane continuum was presented 6] by B. Knaster. Twenty-five years later Moise [11 constructed an hereditarily equivalent and hereditarily indecomposable plane continuum, and called it a pseudo-arc. Further, as a consequence of Bing's [3] characterization of the pseudo-arc, it turned out to be topologically equivalent to the Knaster curve. Moise's result was a starting point to intensive research on this very special continuum by a number of authors (see the survey paper [8]). In this paper we refer to some investigations made by Knaster before Moise's construction. Among the results obtained by Knaster during World War II one can find the following announcement, originally presented in Kiev in 1940:

There exists a real-valued, monotone mapping from the plane that is not constant on any arc.

In the construction Knaster's hereditarily indecomposable continua were exploited. Actually, Knaster's result can be reformulated in the following stronger version (see [10], p. 225):

There exists a real valued, monotone mapping from the plane such that all pointinverses are hereditarily indecomposable.

Unfortunately, Knaster's notes concerning this result were burned during the war, Knaster had never written down the result again, and even his closest exstudents do not know his original idea of construction.

A result similar to that announced by Knaster (with higher dimensional analogues) was proved by Brown [5] in 1958. In fact, a continuous decomposition into hereditarily indecomposable continua of each Euclidean $n$-space with one point deleted was constructed, such that the real line was the quotient space.

Having no confidence that we follow Knaster's idea, in this paper we construct an example of an open mapping as in the announcement. Then we use it to obtain a continuous decomposition of the plane band (annulus) into pseudo-arcs, such that

Received by the editors May 25, 1994 and, in revised form, February 3, 1998.

1991 Mathematics Subject Classification. Primary 54F15; Secondary 54F50, 54B15.

Key words and phrases. continuous decomposition, continuum, plane, pseudo-arc.

(C)2000 American Mathematical Society 
the quotient space is the real line (simple closed curve). This result was announced [1] by Anderson in 1950, but no proof was forthcoming.

In 1959 Bing and Jones [4] constructed a plane circularly chainable curve with a continuous decomposition into pseudo-arcs and with a simple closed curve as the quotient space. They called it a circle of pseudo-arcs and proved its homogeneity. As a subspace of this curve a chainable arc of pseudo-arcs was obtained. Ten years ago Lewis [7] asked whether each curve having a continuous decomposition into pseudo-arcs with an arc as the quotient space is topologically equivalent to that constructed by Bing and Jones. In the present paper we derive some new plane curves from our construction: a new "arc of pseudo-arcs" and a new "circle of pseudo-arcs" (they are triodic and thus topologically different from the curves constructed by Bing and Jones). This answers the question of Lewis in the negative.

\section{PRELIMINARIES}

Spaces are metric and mappings are continuous in this paper. The open $\varepsilon$ neighborhood around a set (a point) $A$ is denoted by $N_{\varepsilon}(A)$.

The symbol dist stands for the usual supremum distance for mappings. Only the uniform convergence is considered for mappings. A mapping $f: X \rightarrow Y$ is called an $\varepsilon$-pushing, provided $X, Y$ are subspaces of some space $U$ and $d(x, f(x))<\varepsilon$ for each $x \in X$. The letters $\mathcal{R}, \mathcal{Z}, \mathcal{C}$ stand for the sets of all real, integer and complex numbers, respectively. The symbols $\bar{u}, \bar{v}, \bar{w}$, etc., denote vectors. If $p$ is a point of a

space $X$, and $X$ admits translation with vector $\bar{u}$, then $p+\bar{u}$ means the image of $p$ by this translation.

The symbols $\overrightarrow{p q}, \overline{p q}, p q$ denote the vector from $p$ to $q$, the straight line segment between $p$ and $q$, and any arc with $p$ and $q$ as its end-points, respectively.

The space of all subcontinua of a space $X$ with the Hausdorff metric is denoted by $C(X)$.

A closed set $T$ in a space $X$ is called terminal in $X$, if either $T \subset K$ or $K \subset T$ for each closed connected set $K \subset X$ with $K \cap T \neq \emptyset$. If each closed connected subset of a connected space $X$ is terminal, then $X$ is said to be hereditarily indecomposable. A connected space $X$ is said to be arc-like if for any $\varepsilon>0$ there exists a mapping $f: X \rightarrow[0,1]$ with $\operatorname{diam} f^{-1}(y)<\varepsilon$ for each $y \in[0,1]$. Note that the last two notions are generalizations of the well-known concepts normally used for continua.

Let $\mathcal{D}$ be an upper semi-continuous decomposition of a compact space $X$. If $X$ admits a retraction $f: X \rightarrow Y=f(X) \subset X$ such that $f^{-1}(y) \in \mathcal{D}$ for each $y \in Y$, then $Y$ is called a continuous selector of $\mathcal{D}$.

\section{Geometric Constructions}

In the subsequent investigation we use the following notion. For any $\varepsilon>0$ we say that a connected space $X$ has $\varepsilon$-herindpro ( $\varepsilon$-hereditary indecomposability property) provided that, for all closed connected sets $K, L \subset X$, if $K \cap L \neq \emptyset$, then either $K \subset N_{\varepsilon}(L)$, or $L \subset N_{\varepsilon}(K)$. The following lemma will be used in the main construction.

Lemma 1. Let a subcontinuum $X$ of a metric space $U$ be the union of two continua $X_{1}, X_{2}$ such that $X_{1}$ and $X_{2}$ each has $\varepsilon$-herindpro, and there is a $\delta$-pushing $f$ : $X \rightarrow p q$ for some arc $p q \subset U$, such that $X_{1} \cap X_{2} \subset f^{-1}(p)$. Then $X$ has $(\varepsilon+2 \delta)$ herindpro. 
Proof. Let $X, X_{1}, X_{2}, p q, f$ be as in the assumptions of the lemma, and let $K$, $L$ be subcontinua of $X$ with $K \cap L \neq \emptyset$. If $L \subset K$, or $K \subset L$, or $L, K \subset X_{1}$, or $L, K \subset X_{2}$, the conclusion is obvious.

If $K \cap f^{-1}(p) \neq \emptyset \neq L \cap f^{-1}(p)$, then either $f(K) \subset f(L)$, or $f(L) \subset f(K)$, and thus $K \subset N_{2 \delta}(L)$,or $L \subset N_{2 \delta}(K)$.

Assume $K \cap f^{-1}(p)=\emptyset$ and $L \cap f^{-1}(p) \neq \emptyset$. Then either $K \subset X_{1}-X_{2}$, or $K \subset X_{2}-X_{1}$. Assume $K \subset X_{1}-X_{2}$. Further assume $K-N_{(\varepsilon+2 \delta)}(L) \neq \emptyset$. Then there is a continuum $L_{1} \subset L \cap X_{1}$ such that $L_{1} \cap K \neq \emptyset \neq L_{1} \cap f^{-1}(p)$. Since $X_{1}$ has $\varepsilon$-herindpro, we have $L_{1} \subset N_{\varepsilon}(K)$. Considering $K \cup L_{1}$ and $L$, we have the previous case. Hence $L \subset N_{2 \delta}\left(K \cup L_{1}\right) \subset N_{(2 \delta+\varepsilon)}(K)$. The other cases are similar.

Considering the representation of the pseudo-arc as the inverse limit of arcs, in the next lemma we observe the existence of arbitrarily large arcs with $\varepsilon$-herindpro for arbitrarily small $\varepsilon$.

Lemma 2. For any $M>0$ and any $\varepsilon>0$ there are $a \delta>0$ and a mapping $\lambda:[0, \delta] \rightarrow[0, M]$ such that

(1) $\lambda(0)=0, \lambda(\delta)=M$, and

(2) the graph $\{(x, \lambda(x)): x \in[0, \delta]\}$ has $\varepsilon$-herindpro.

Remark. Actually, $\delta$ can be arbitrarily chosen from some interval $\left(0, \delta_{0}\right)$.

Proof. To make the lemma evident, it suffices to consider a representation

$$
P=\lim _{\leftarrow}\left(I_{n}, f_{n}\right)
$$

of the pseudo-arc $P$ as the inverse limit with $I_{n}=[0,1]$, and to take $f_{n, m}=$ $f_{m} \circ f_{m+1} \circ \ldots \circ f_{n}: I_{n+1} \rightarrow I_{m}$ for suitably large $m$ and $n$. Then the mapping $\lambda(x)=M \cdot f_{n, m}(x / \delta)$ with suitably small $\delta$ satisfies the conclusion of the lemma by the hereditary indecomposability of $P$. The details are left to the reader.

In our further investigations we consider the space

$$
\mathcal{S}=\mathcal{X} \times \mathcal{R}
$$

where $\mathcal{R}$ is the set of all reals, and either $\mathcal{X}=\mathcal{R}$, or $\mathcal{X}$ is the circle $S^{1}=\{x \in \mathcal{C}$ : $|x|=1\}$, with the convex metric $d_{1}(p, q)=\min \left\{\right.$ length $p q: p q$ is an $\operatorname{arc}$ in $\left.S^{1}\right\}$, for $p, q \in S^{1}$. If $\mathcal{S}=\mathcal{R}^{2}$ we consider the usual Euclidean metric in $\mathcal{S}$. If $\mathcal{S}=S^{1} \times \mathcal{R}$, then let $d((x, y),(u, w))=\sqrt{\left(d_{1}(x, u)\right)^{2}+|y-w|^{2}}$, for $x, u \in S^{1}$ and $y, w \in \mathcal{R}$. We consider the usual vector space acting on $\mathcal{S}$. In particular, if $\mathcal{S}=S^{1} \times \mathcal{R}$ and $\bar{v}=\langle a, b\rangle$ is a vector with coordinates $a, b \in \mathcal{R}$, then for any $p=(x, y) \in \mathcal{S}$ we have $p+\bar{v}=(x \cdot z, y+b)$, where $z=(\cos a, \sin a)$ and $x \cdot z$ is the usual product of the complex numbers $x, z$.

We will use the term stable mapping on $\mathcal{S}$ in the following stronger meaning, although it differs from that commonly used for the term. A mapping $f: \mathcal{S} \rightarrow \mathcal{S}$ is said to be stable provided there is a bounded set $U \subset \mathcal{S}$ such that $f(x)=x$ for each $x \in \mathcal{S}-U$. Two decompositions $\mathcal{D}_{1}$ and $\mathcal{D}_{2}$ of $\mathcal{S}$ are said to be stably equivalent, if there is a stable homeomorphism $h: \mathcal{S} \rightarrow \mathcal{S}$ such that $h(D) \in \mathcal{D}_{2}$ for each $D \in \mathcal{D}_{1}$.

Let $f: X \rightarrow X$ be a surjection, and $\mathcal{D}_{1}, \mathcal{D}_{2}$ be decompositions of the space $X$. We say that $f$ transforms $\mathcal{D}_{1}$ onto $\mathcal{D}_{2}$, if $f^{-1}(D) \in \mathcal{D}_{1}$ for each $D \in \mathcal{D}_{2}$.

Fix the following decomposition of $\mathcal{S}$ :

$$
\mathcal{D}_{0}=\{\{x\} \times \mathcal{R}: x \in \mathcal{X}\} .
$$


The next theorem is crucial in this paper. Applying the theorem, a series of decompositions of the plane or open annulus into lines will be constructed. These will be stably equivalent to the decomposition into vertical lines or radial segments so that each element can be continuously compactified by adding points at the top and bottom, or inside and outside. This sequence of compactified decompositions will converge to the desired decomposition into pseudo-arcs.

Theorem 3. For each bounded set $U \subset \mathcal{S}$ and for each $M>\operatorname{diam} U$ there are a decomposition $\mathcal{G}$ of $\mathcal{S}$ stably equivalent to $\mathcal{D}_{0}$ and a stable surjection $g: \mathcal{S} \rightarrow \mathcal{S}$ such that

(1) $g$ transforms the decomposition $\mathcal{G}$ onto $\mathcal{D}_{0}$,

(2) $g$ is a (1/M)-pushing, and

(3) for each $G \in \mathcal{G}$ and for each continuum $C \subset U \cap G$ the continuum $C$ has $(1 / M)$-herindpro.

Remark. Observe that the property described in the theorem is invariant with respect to stable homeomorphisms in the sense that, for each homeomorphism $h: \mathcal{S} \rightarrow \mathcal{S}$, if a decomposition $\mathcal{D}=\left\{h(D): D \in \mathcal{D}_{0}\right\}$ has the property, then each decomposition $\mathcal{D}^{\prime}$ which is stably equivalent to $\mathcal{D}$ has the property, too. In particular, it holds for $\mathcal{D}=\mathcal{D}_{0}$.

Proof of Theorem 3 . The idea of the proof is the following (see Figure1). First, we construct a homeomorphism $h_{1}: \mathcal{S} \rightarrow \mathcal{S}$ such that for each straight line $D \in \mathcal{D}_{0}$ its image lies close to $D$ (in the sense that the Hausdorff distance between them is small enough). In this construction the conclusion of Lemma2 2 is employed to assure local $(1 / M)$-herindpro for $h_{1}(D)$. Second, we modify $h_{1}$ to a homeomorphism $h: \mathcal{S} \rightarrow \mathcal{S}$ satisfying $h(x)=x$ for each $x$ in the complement of a bounded neighborhood of $U$. Then we obtain the required decomposition $\mathcal{G}=\left\{h(D): D \in \mathcal{D}_{0}\right\}$. Finally, we take a mapping $g: \mathcal{S} \rightarrow \mathcal{S}$ such that for each $D \in \mathcal{D}_{0}$ the partial mapping $g \mid h(D)$ is the orthogonal projection of $h(D)$ onto $D$.

Let $D_{1}=\{(x, y) \in S: x=1\}$ and note that $D_{1} \in \mathcal{D}_{0}$. Fix $\sigma, \varepsilon<1$ such that $0<\sigma<\varepsilon / 2<\varepsilon<1 / M$, and let $\bar{u}=\langle 0,2 M\rangle, \bar{v}=\langle\sigma, 2 M\rangle, \bar{v}_{0}=(1 /|\bar{v}|) \bar{v}$. Define $\phi$ as the angle between the vectors $\bar{u}$ and $\bar{v}$.

For $\varepsilon / 2$ and $2 M$ take a $\delta$ and a $\lambda:[0, \delta] \rightarrow[0,2 M]$ guaranteed by Lemma 2] assuming (see the remark after Lemma 2)

$$
\delta<\varepsilon / 4 \text { and } \delta<M \sin ^{2} \phi
$$

and, if $\mathcal{X}=S^{1}$, we add the following extra assumption on $\delta$ :

(3.1) For any point $p \in \mathcal{S}$ and for the spiral line $L(p)=\{p+t \bar{v}: t \in \mathcal{R}\}$, if $r_{1}$ and $r_{2}$ are two consecutive points of the intersection $L \cap D_{1}$, then the number $\left(d\left(r_{1}, r_{2}\right) \cdot \sin \phi\right) / 2 \delta$ is a fixed integer $j$.

Now we fix two sequences of points $p_{k}, q_{k} \in D_{1}$ defined by

$$
\begin{gathered}
p_{k}=(1,2 k \cdot \delta / \sin \phi) \in \mathcal{S}, \\
q_{k}=(1,(2 k+1) \cdot \delta / \sin \phi) \in \mathcal{S}, \text { for each } k \in \mathcal{Z} .
\end{gathered}
$$

Observe that, if $\mathcal{S}=S^{1} \times \mathcal{R}$, by virtue of (3.1) we have

$$
\begin{aligned}
& L\left(p_{k}\right) \cap D_{0}=\left\{p_{k+m j}: m \in \mathcal{Z}\right\}, \\
& L\left(q_{k}\right) \cap D_{0}=\left\{q_{k+m j}: m \in \mathcal{Z}\right\} .
\end{aligned}
$$


A CONTINUOUS CIRCLE OF PSEUDO-ARCS FILLING UP THE ANNULUS

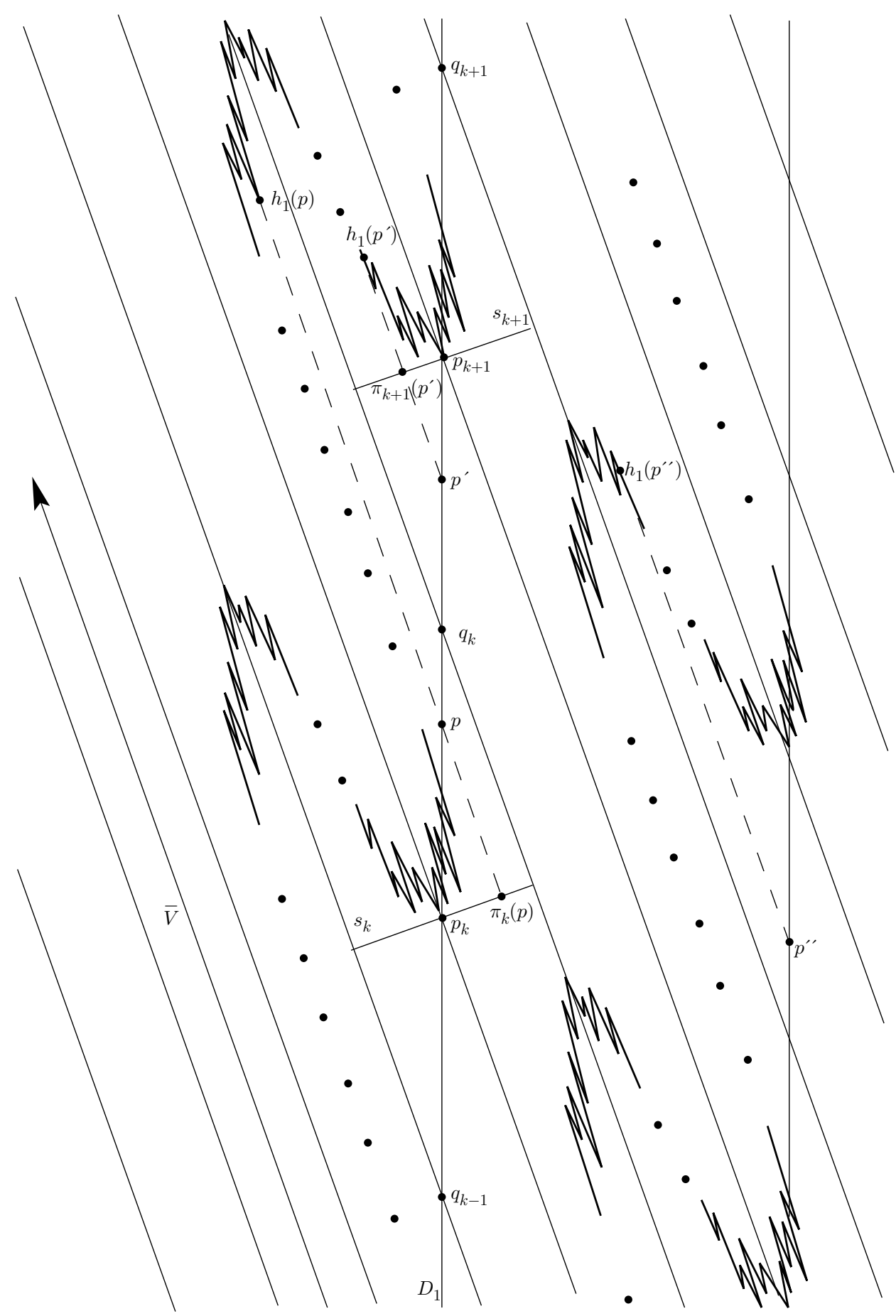

Figure 1. 
Let $s_{k}$ denote the segment in $\mathcal{S}$ perpendicular to $\bar{v}$, with length $2 \delta$, having $p_{k}$ as its midpoint. Let $\pi_{k}$ be the orthogonal projection of the segment $\overline{q_{k-1} q_{k}} \subset D_{1}$ onto $s_{k}$. For any $p \in \overline{p_{k} q_{k}}$ let

$$
h_{1}(p)=\pi_{k}(p)+\lambda\left(d\left(p_{k}, \pi_{k}(p)\right)\right) \cdot \bar{v}_{0} .
$$

Next, let $\mu=(M-\delta \cdot \cot \phi) / M$, and observe that

$$
1>\mu=(M-\delta \cdot \cot \phi) / M>\left(M-M \cdot \sin ^{2} \phi \cdot \cot \phi\right) / M=1-(\sin 2 \phi) / 2 \geq 1 / 2 .
$$

Then, for any $p \in \overline{q_{k} p_{k+1}}$ let

$$
h_{1}(p)=\pi_{k+1}(p)+\mu \lambda\left(d\left(\pi_{k+1}(p), p_{k+1}\right)\right) \bar{v}_{0} .
$$

Observe that $h_{1}(p)$ is well defined for all points $p$ in $D_{1}$.

Next, note that for a fixed $k$ the set $\left\{h_{1}(p): p \in \overline{p_{k} q_{k}}\right\}$ is isometric to the graph of $\lambda$, and the set $\left\{h_{1}(p): p \in \overline{q_{k} p_{k+1}}\right\}$ is isometric to the graph of $\mu \cdot \lambda$. Therefore both these sets have $\varepsilon$-herindpro.

Take any continuum $K$ in $h_{1}\left(D_{1}\right)$ with diam $K<M$. Since each of diam $h_{1}\left(\overline{p_{k} q_{k}}\right)$ and diam $h_{1}\left(\overline{q_{k} p_{k+1}}\right)$ is greater than $M$ for all $k$, there is a $k$ such that either $K \subset$ $h_{1}\left(\overline{p_{k} p_{k+1}}\right)$, or $K \subset h_{1}\left(\overline{q_{k} q_{k+1}}\right)$. Assume $K \subset h_{1}\left(\overline{p_{k} p_{k+1}}\right)$ (the other case is similar). The set $h_{1}\left(\overline{p_{k} p_{k+1}}\right)=h_{1}\left(\overline{p_{k} q_{k}}\right) \cup h_{1}\left(\overline{q_{k} p_{k+1}}\right)$ admits a $\delta$-pushing onto the segment $\overline{\pi_{k}\left(q_{k}\right) h_{1}\left(q_{k}\right)}$ (the orthogonal projection), and the $\operatorname{arcs} h_{1}\left(\overline{p_{k} q_{k}}\right)$ and $h_{1}\left(\overline{q_{k} p_{k+1}}\right)$ have $(\varepsilon / 2)$-herindpro. This implies that $h_{1}\left(\overline{p_{k} p_{k+1}}\right)$ has $(1 / M)$-herindpro by Lemma 1 (we have $(\varepsilon / 2)+2 \delta<(\varepsilon / 2)+2(\varepsilon / 4)=\varepsilon<1 / M)$, and thus $K$ also has $(1 / M)$-herindpro. Hence all continua in $h_{1}\left(D_{1}\right)$ with diameters $<M$ have $(1 / M)$ herindpro.

To define $h_{1}$ for each $p \in \mathcal{S}$ take the projection $p^{\prime}$ of $p$ into $D_{1}$ in the direction of $\bar{v}$, and let

$$
h_{1}(p)=p+\overrightarrow{p^{\prime} h_{1}\left(p^{\prime}\right)} .
$$

Comment. If $\mathcal{X}=S^{1}$ the projection is not uniquely determined. However, if $p^{\prime}$ and $p^{\prime \prime}$ are such projections, their distance is a multiple of $2 \delta / \sin \phi($ see $(3.1))$. Then, from the definition of $h_{1}(q)$ for $q \in D_{1}$, it follows that $\overrightarrow{p^{\prime} h_{1}\left(p^{\prime}\right)}=\overrightarrow{p^{\prime \prime} h_{1}\left(p^{\prime \prime}\right)}$. This implies the unique determination of $h_{1}$.

The mapping $h_{1}$ is a uniformly continuous homeomorphism of $\mathcal{S}$ onto itself. Moreover, for all $D \in \mathcal{D}_{0}$ the sets $h_{1}(D)$ are mutually isometric, and thus for each continuum $K$ contained in any such $h_{1}(D)$ with diam $K<M$, the continuum $K$ has $(1 / M)$-herindpro.

Now we modify $h_{1}$ to make it stable. Take $\alpha>0$ large enough that $U \subset N_{\alpha}\left(p_{0}\right)$. Let $\beta=|\bar{v}|$ and observe that $\left|\overrightarrow{p h_{1}(p)}\right| \leq \beta$ for each $p \in \mathcal{S}$. Further, define $\gamma:[0, \infty) \rightarrow[0,1]$ by

$$
\gamma(x)= \begin{cases}1 & \text { for } \quad x \in[0, \alpha+\beta] \\ \frac{\alpha+3 \beta-x}{2 \beta} & \text { for } \quad x \in[\alpha+\beta, \alpha+3 \beta], \\ 0 & \text { for } \quad x>\alpha+3 \beta\end{cases}
$$

If $\mathcal{X}=\mathcal{R}$, for any $p \in \mathcal{S}$ let

$$
h(p)=p+\gamma\left(d\left(p, p_{0}\right)\right) \overrightarrow{p h_{1}(p)},
$$


and if $\mathcal{X}=S^{1}$, for any $p=(x, y) \in \mathcal{S}$ let

$$
h(p)=p+\gamma(|x|) \overrightarrow{p h_{1}(p)} .
$$

One can verify that in both cases $h$ is a stable homeomorphism.

Define $\mathcal{G}=\left\{h(D): D \in \mathcal{D}_{0}\right\}$. Let a continuum $K \subset U$ be contained in an element $h(D)$ of $\mathcal{G}$. Since the mappings $h_{1}, h$ coincide on $N_{\alpha+\beta}\left(p_{0}\right)$ and $h^{-1}(U) \subset$ $N_{\alpha+\beta}\left(p_{0}\right)$, we have $K \subset h_{1}(D)$. Further, $\operatorname{diam} K \leq \operatorname{diam} U<M$, and thus $K$ has $\varepsilon$-herindpro.

For any $p=(x, y) \in \mathcal{S}$ let $\left(x_{p}, y_{p}\right)=h^{-1}(p)$. Define $g: \mathcal{S} \rightarrow \mathcal{S}$ by

$$
g(p)=\left(x_{p}, y\right) .
$$

Then $g$ is a $(1 / M)$-pushing such that for each $D \in \mathcal{D}_{0}$ we have $g^{-1}(D)=h(D) \in$ $\mathcal{G}$, and $g$ is stable. The proof of Theorem 3 is complete.

\section{TOPOLOGICAL RESULTS}

In the main construction we employ the following theorem on inverse limits. The idea of this theorem resembles that of a theorem by Anderson and Choquet [2] (Theorem 1, p.348).

Theorem 4. Let $X$ be a complete metric space and let a sequence $f_{n}: X \rightarrow X$ of surjections $f_{n}$ satisfy the following two conditions:

(a) $\sum_{n=1}^{\infty} \operatorname{dist}\left(f_{n}, \operatorname{id}_{X}\right)<\infty$.

(b) For each positive integer $k$ the sequence $f_{k}^{n+k}=f_{k} \circ f_{k+1} \circ \ldots \circ f_{k+n-1}$ converges uniformly.

Then the function $h: X \rightarrow \lim _{\longleftarrow}\left(X, f_{n}\right)$ defined by

$$
h(x)=\left(\lim f_{1}^{1+n}(x), \lim f_{2}^{2+n}(x), \ldots\right)
$$

is a homeomorphism, and

$$
h^{-1}\left(x_{1}, x_{2}, \ldots\right)=\lim x_{n}
$$

for each $\left(x_{1}, x_{2}, \ldots\right) \in \lim _{\leftarrow}\left(X, f_{n}\right)$.

Proof. For any $x \in X$ let $h(x)=\left(x_{1}, x_{2}, \ldots\right)$. Since

$$
\begin{aligned}
f_{k-1}\left(x_{k}\right) & =f_{k-1}\left(\lim _{n} f_{k}^{k+n}(x)\right)=f_{k-1}\left(\lim _{n} f_{k} \circ f_{k-1} \circ \ldots \circ f_{k+n-1}(x)\right) \\
& =\lim _{n} f_{k-1} \circ f_{k} \circ f_{k+1} \circ \ldots \circ f_{k+n-1}(x)=x_{k-1},
\end{aligned}
$$

$h$ is well defined.

On the other hand, for any $\left(y_{1}, y_{2}, \ldots\right) \in \lim \left(X, f_{n}\right)$, observe that $y_{n}$ converges to some $y$ by condition (a). For any $k$ we have $y_{k}=f_{k}^{k+m}\left(y_{k+m}\right) \stackrel{m}{\rightarrow} \lim _{n} f_{k}^{k+n}(y)$ by condition (b), and thus $\left(y_{1}, y_{2}, \ldots\right)=h(y)$. Hence $h$ is a one-to-one mapping from $X$ onto $\lim _{\longleftarrow}\left(X, f_{n}\right)$. The continuity of $h\left(\right.$ of $\left.h^{-1}\right)$ can be proven by condition (b) (by condition (a), respectively). The details are left to the reader.

The following theorem (in a weaker version; see the introduction) was announced by Knaster in 1940 [10], but the proof has neither been presented, nor published. In this theorem we obtain a continuous decomposition of the plane into "pseudo-lines", i.e., closed connected sets, each admitting a pseudo-arc as its 2-point compactification. These pseudo-arcs are irreducible between the two points of the compactification. 
Theorem 5 (Knaster). There exists a continuous decomposition $\mathcal{D}$ of the plane $\mathcal{R}^{2}$ into closed, connected, noncompact, hereditarily indecomposable, arc-like sets, such that the quotient space is homeomorphic to the real line.

Moreover, for each $\varepsilon>0$ the decomposition $\mathcal{D}$ can be constructed to admit a surjective $\varepsilon$-pushing $f: \mathcal{R}^{2} \rightarrow \mathcal{R}^{2}$ transforming $\mathcal{D}$ onto $\mathcal{D}_{0}$.

Proof. Fix any $\varepsilon>0$ and let $U_{n}=[-n, n]^{2} \subset \mathcal{R}^{2}$. Applying Theorem [3, we construct a proper sequence $\mathcal{D}_{0}, \mathcal{D}_{1}, \ldots$ of decompositions of $\mathcal{R}^{2}$ and a sequence $g_{n}: \mathcal{R}^{2} \rightarrow \mathcal{R}^{2}$ of surjective $\left(\varepsilon / 2^{n}\right)$-pushings transforming $\mathcal{D}_{n}$ onto $\mathcal{D}_{n-1}$, such that for each $k$ the sequence $g_{k}^{k+n}=g_{k} \circ g_{k+1} \circ \ldots \circ g_{k+n-1}$ converges as $n \rightarrow \infty$. More precisely, the mappings $g_{k}$ are chosen such that

(5.1) $\operatorname{dist}\left(g_{k}^{k+n}, g_{k}^{k+n+1}\right) \leq \varepsilon / 2^{n+k}$ for all $k$ and $n$.

Then we apply Theorem 4 to obtain the conclusion.

As previously, take $\mathcal{D}_{0}=\{\{x\} \times \mathcal{R}: x \in \mathcal{R}\}$, and let $M_{0}=\max \{4,2 / \varepsilon\}$. Then take a decomposition $\mathcal{D}_{1}$ and a mapping $g_{1}: \mathcal{R}^{2} \rightarrow \mathcal{R}^{2}$ guaranteed by Theorem 3 for $\mathcal{D}_{0}, U_{1}$ and $M_{0}$.

Assume that we have constructed decompositions $\mathcal{D}_{0}, \ldots, \mathcal{D}_{i}$, stable mappings $g_{1}, \ldots, g_{i}: \mathcal{R}^{2} \rightarrow \mathcal{R}^{2}$, and numbers $M_{0}, \ldots, M_{i-1}$ such that

(5.2) $\mathcal{D}_{j}$ is stably equivalent to $\mathcal{D}_{0}$ for $j \in\{1, \ldots, i\}$,

(5.3) $M_{j} \geq \max \left\{4 j, 2^{j+1} / \varepsilon\right\}$ for $j \in\{0, \ldots, i-1\}$,

(5.4) $g_{j}$ is a $\left(1 / M_{j-1}\right)$-pushing for $j \in\{1, \ldots, i\}$,

(5.5) condition (5.1) is satisfied for $n+k \leq i$,

(5.6) $g_{j}$ transforms $\mathcal{D}_{j}$ onto $\mathcal{D}_{j-1}$ for $j \in\{1, \ldots, i\}$, and

(5.7) for each $D \in \mathcal{D}_{j}$ and for each continuum $C \subset D \cap U_{j}$, the continuum has $\left(1 / M_{j-1}\right)$-herindpro for $j \in\{1, \ldots, i\}$.

Assume $n+k=i+1$. Let $\delta(n, k)>0$ be a number such that if $d(p, q)<\delta(n, k)$, then $d\left(g_{k}^{k+n}(p), g_{k}^{k+n}(q)\right)<\varepsilon / 2^{n+k}$. Let $M_{i}=\max \left(\left\{2^{i+1} / \varepsilon\right\} \cup\{1 / \delta(n, k): k+n=\right.$ $i+1\})$. Then take a decomposition $\mathcal{D}_{i+1}$ and a mapping $g_{i+1}: \mathcal{R}^{2} \rightarrow \mathcal{R}^{2}$ guaranteed by Theorem 3 (see also the remark after Theorem 3) for $\mathcal{D}_{i}, U_{i+1}$ and $M_{i}$. One can verify that conditions (5.2)-(5.7) are satisfied if $i$ is replaced by $i+1$.

By the induction we obtain sequences $M_{n}, g_{n}, \mathcal{D}_{n}$ satisfying (5.2)-(5.7) for all $i$. Applying Theorem 4 observe that $\lim _{\leftarrow}\left(\mathcal{R}^{2}, g_{n}\right)$ is homeomorphic to $\mathcal{R}^{2}$ and the mapping $h\left(x_{1}, x_{2}, \ldots\right)=\lim x_{n}$ is a homeomorphism between these spaces. Let us identify these spaces by this mapping.

Next, let $f_{n}: \lim \left(\mathcal{R}^{2}, g_{n}\right) \rightarrow \mathcal{R}^{2}$ be the projections of this inverse system. Observe that $f_{n}$ is $\left(\varepsilon / 2^{n-1}\right)$-pushing. Indeed, let $\left(x_{1}, x_{2}, \ldots\right)$ be a point of the inverse limit identified with $x_{0}=\lim x_{n}$. Then $f_{n}\left(x_{0}\right)=x_{n}$, and $d\left(f_{n}\left(x_{0}\right), x_{0}\right) \leq$ $\sum_{n}^{\infty} d\left(x_{k}, x_{k+1}\right)<\sum_{n}^{\infty} \varepsilon / 2^{k}=\varepsilon / 2^{n-1}$. Let

$$
\mathcal{D}_{\infty}=\left\{f_{1}^{-1}(D): D \in \mathcal{D}_{0}\right\}
$$

and observe that

$$
\mathcal{D}_{\infty}=\left\{f_{n}^{-1}(D): D \in \mathcal{D}_{n-1}\right\}
$$

for each $n$.

Further, note that $\mathcal{D}_{\infty}$ is a decomposition of $\mathcal{R}^{2}$ such that for each $D \in \mathcal{D}_{\infty}$ there are $D_{n} \in \mathcal{D}_{n}$ satisfying $g_{n}\left(D_{n}\right)=D_{n-1}$ and

$$
D=\lim _{\longleftarrow}\left(D_{n}, g_{n+1} \mid D_{n+1}\right) \text {. }
$$


The sets $D_{n}$ are closed in $\mathcal{R}^{2}$ and homeomorphic to the real line. Thus $D$ is connected, closed and arc-like.

Let $L, K$ be arbitrary subcontinua of some $D \in \mathcal{D}_{\infty}$ with $L \cap K \neq \emptyset$. We have $f_{n}(L) \cup f_{n}(K) \subset D_{n-1} \cap U_{n-1}$ for sufficiently large $n$ and some $D_{n-1} \in \mathcal{D}_{n-1}$. Then either $f_{n}(L) \subset N_{\alpha(n)}\left(f_{n}(K)\right)$ for infinitely many $n$, or $f_{n}(K) \subset N_{\alpha(n)}\left(f_{n}(L)\right)$ for infinitely many $n$, where $\alpha(n)=\varepsilon / 2^{n-1}$. Since $\lim f_{n}=\operatorname{id}_{\mathcal{R}^{2}}$, it follows that either $L \subset K$, or $K \subset L$.

Assume that $L, K \subset D$ are closed (not necessarily compact), connected sets with $L \cap K \neq \emptyset$. Then there are continua $L_{n}, K_{n}$ approximating $L, K$ (respectively) such that $L_{n} \subset L, K_{n} \subset K$ and $L_{n} \cap K_{n} \neq \emptyset$. We have either $L_{n} \subset K_{n}$ for infinitely many $n$, or $K_{n} \subset L_{n}$ for infinitely many $n$. So, again we infer that either $L \subset K$, or $K \subset L$. Hence $D$ is hereditarily indecomposable for each $D \in \mathcal{D}_{\infty}$.

Finally, each $\mathcal{D}_{n}$ is continuous and $f_{n}$ is an $\left(\varepsilon / 2^{n-1}\right)$-pushing. Thus $\mathcal{D}_{\infty}$ is continuous as well. We take $f_{1}$ for the required mapping $f$. The proof is then complete.

Remark. Theorem 15 remains true, if we replace $\mathcal{R}^{2}$ by $S^{1} \times \mathcal{R}$. The argument is the same.

Remetrizing $\mathcal{R}^{2}$ properly, we can identify $\mathcal{R}^{2}$ with its subset $\mathcal{B}_{0}=\left\{(x, y) \in \mathcal{R}^{2}\right.$ : $|y|<1\}$ such that $\mathcal{D}_{0}=\{\{x\} \times(-1,1): x \in \mathcal{R}\}$. Define $\mathcal{B}=\left\{(x, y) \in \mathcal{R}^{2}:|y| \leq 1\right\}$. Then we can extend the mappings $g_{n}: \mathcal{B}_{0} \rightarrow \mathcal{B}_{0}$ used in the proof of Theorem 5 to mappings $g_{n}^{*}: \mathcal{B} \rightarrow \mathcal{B}$ by letting $g_{n}^{*}(x)=x$ for $x \in \mathcal{B}-\mathcal{B}_{0}$ (the $g_{n}$ 's are stable on $\left.\mathcal{R}^{2}\right)$. Next, extend the decompositions $\mathcal{D}_{n}$ of $\mathcal{B}_{0}$ to decompositions $\mathcal{D}_{n}^{*}$ of $\mathcal{B}$ adding the proper pair of points $(x,-1),(x, 1)$ to each element $D$ of $\mathcal{D}_{n}$ to compactify it to an arc (the $\mathcal{D}_{n}$ 's are stably equivalent to $\mathcal{D}_{0}$ in $\mathcal{R}^{2}$ ). Then, similarly as in the proof of Theorem [5] we obtain a decomposition $\mathcal{D}_{\infty}^{*}$ of $\mathcal{B}$. The elements of $\mathcal{D}_{\infty}^{*}$ are arc-like and hereditarily indecomposable; thus they are pseudo-arcs [3]. We have obtained the following theorem.

Theorem 6. There exists a continuous decomposition $\mathcal{D}$ of the plane band $\mathcal{B}=$ $\left\{\left(x, y \in \mathcal{R}^{2}:|y| \leq 1\right\}\right.$ into pseudo-arcs, such that:

(1) the quotient space is homeomorphic to the real line $\mathcal{R}$, and

(2) each pseudo-arc $D \in \mathcal{D}$ intersects the line $y=1$ at exactly one point $p_{D}$, and the line $y=-1$ at exactly one point $q_{D}$, and $D$ is irreducible between $p_{D}$ and $q_{D}$. (Each of these lines yields a continuous selection of the decomposition.)

Moreover, for each $\varepsilon>0$ the decomposition $\mathcal{D}$ can be constructed to admit a surjective $\varepsilon$-pushing $f: \mathcal{B} \rightarrow \mathcal{B}$ transforming $\mathcal{D}$ onto $\mathcal{D}_{0}^{*}$.

Applying the modification of Theorem 5 concerning $S^{1} \times \mathcal{R}$ (see the remark after Theorem [5), similarly as the above theorem, we obtain the decomposition as in the title of the paper.

Theorem 7. There exists a decomposition $\mathcal{D}$ of the annulus $\mathcal{A}=\{z \in \mathcal{C}: 1 \leq$ $|z| \leq 2\}$ into pseudo-arcs, such that:

(1) the quotient space is a simple closed curve, and

(2) each pseudo-arc $D \in \mathcal{D}$ intersects the circle $C_{1}=\{z \in \mathcal{C}:|z|=1\}$ at exactly one point $p_{D}$, and the circle $C_{2}=\{z \in \mathcal{C}:|z|=2\}$ at exactly one point $q_{D}$, and $D$ is irreducible between $p_{D}$ and $q_{D}$. (Each of these circles yields a continuous selection of $\mathcal{D}$.) 
Moreover, for each $\varepsilon>0$ the decomposition $\mathcal{D}$ can be constructed to admit a surjective $\varepsilon$-pushing of $\mathcal{A}$ transforming $\mathcal{D}$ onto the decomposition $\mathcal{D}_{0}^{*}$ of $\mathcal{A}$ into maximal radial segments.

Let $\mathcal{D}$ be the decomposition as in the above theorem. Without loss of generality assume that the points $p_{D}, q_{D}$ are the ends of maximal radial segments in $\mathcal{A}$. Define $f_{1}: \mathcal{A} \rightarrow C_{1}$ by $f_{1}(z)=p_{D}$ for $z \in D \in \mathcal{D}$. Then $f_{1}$ is a monotone, open retraction. Let $\omega: C(\mathcal{A}) \rightarrow[0,1]$ be a Whitney map. Let $a_{0}=\inf \{\omega(D): D \in \mathcal{D}\}$ and take any $a$ with $0<a<a_{0}$. Observe that since the elements of $\mathcal{D}$ are hereditarily indecomposable, for each $z \in \mathcal{A}$ there is exactly one set $P_{z} \in \omega^{-1}(a)$ such that $z \in P_{z} \subset D_{z}$, where $D_{z}$ is the element of $\mathcal{D}$ containing $z$. Thus we obtain a decomposition $\mathcal{P}_{a}=\left\{P_{z}: z \in \mathcal{A}\right\}$ of $\mathcal{A}$ into pseudo-arcs. We show that this decomposition is continuous.

In fact, note that a decomposition $\mathcal{F}$ of a compact space $X$ into subcontinua of $X$ is continuous if and only if $\mathcal{F}$ is a closed subset of the hyperspace $C(X)$. Observe that the set $\mathcal{G}=\{C \in C(\mathcal{A}): C \subset D$ for some $D \in \mathcal{D}\}$ is closed in $C(\mathcal{A})$ by the continuity of $\mathcal{D}$, and that $\omega^{-1}(a)$ is closed in $C(\mathcal{A})$ by the continuity of $\omega$. Since $\mathcal{P}_{a}=\mathcal{G} \cap \omega^{-1}(a)$, it follows that $\mathcal{P}_{a}$ is a closed subset of $C(\mathcal{A})$. So $\mathcal{P}_{a}$ is continuous.

Further, we prove that the quotient space $\mathcal{A} / \mathcal{P}_{a}$ is homeomorphic to $\mathcal{A}$. Indeed, extend the decomposition $\mathcal{P}_{a}$ to $\mathcal{R}^{2}$ taking singletons outside of $\mathcal{A}$. Then the quotient space is homeomorphic to $\mathcal{R}^{2}$ by the well-known Moore theorem [12] on upper semi-continuous decompositions of the plane into non-separating plane continua. Finally, we see that $\mathcal{A} / \mathcal{P}_{a}$ is a subcontinuum of the quotient space with the boundary composed of two disjoint simple closed curves (the images of $C_{1}$ and $C_{2}$ ), and thus $\mathcal{A} / \mathcal{P}_{a}$ is a topological annulus.

Let $g_{a}: \mathcal{A} \rightarrow \mathcal{A}$ be the composition of the quotient map $\mathcal{A} \rightarrow \mathcal{A} / \mathcal{P}_{a}$ and of a homeomorphism $\mathcal{A} / \mathcal{P}_{a} \rightarrow \mathcal{A}$ such that $g_{a}(z)=z$ for each $z \in C_{1} \cup C_{2}$. Let $U=\{z \in \mathcal{C}: 1<|z|<2$ and $0<\arg z<\pi\}$. Since $g_{a}$ is open and monotone, the set $g_{a}^{-1}(U)$ is an open, connected subset of the plane. Note that bd $g_{a}^{-1}(U)$ is the union of four connected sets, namely $\{z \in \mathcal{C}:|z|=j$ and $0 \leq \arg z \leq \pi\}$ and $g_{a}^{-1}(\{z \in \mathcal{A}: \arg z=(j-1) \cdot \pi\})$ for $j \in\{1,2\}$, such that the four points $(0,-2),(0,-1),(0,1)$ and 0,2$)$ join them to form a connected set. Thus the set $g_{a}^{-1}(U)$ is simply connected. It follows that $g_{a}^{-1}(U)$ is homeomorphic to the plane $\mathcal{R}^{2}$. The decomposition $\mathcal{P}_{a}$ restricted to $g_{a}^{-1}(U)$ is a continuous decomposition of $g_{a}^{-1}(U)$ into pseudo-arcs. Thus we have obtained another proof of the Lewis-Walsh theorem saying that the plane admits a continuous decomposition into pseudo-arcs 9.

Even more interesting are the sets $\tilde{C}_{i}=g_{a}^{-1}\left(C_{i}\right), i=1,2$. First, note that they are curves. Indeed, each $g_{a}^{-1}(p)$ has empty interior in $f_{1}^{-1}(p)$, and we have $f_{1}^{-1}(p)-g_{a}^{-1}(p) \subset \mathcal{R}^{2}-\tilde{C}_{1}$. Therefore $\tilde{C}_{i}$ has empty interior in $\mathcal{R}^{2}$, and thus it is one-dimensional for $i \in\{1,2\}$. Next, observe that $\mathcal{P}_{a}$ restricted to $\tilde{C}_{1}$ is a continuous decomposition of $\tilde{C}_{1}$ into pseudo-arcs, and the circle $C_{1}$ is a continuous selector of this decomposition. Similarly, taking any arc $L \subset C_{1}$ instead of $C_{1}$, we obtain the curve $\tilde{L}=g_{a}^{-1}(L) \subset \tilde{C}_{1}$ with the continuous decomposition into pseudo-arcs and with the continuous selector $L$ of this decomposition. Any curve having a continuous decomposition into pseudo-arcs with an arc (a simple closed curve) as a continuous selector of the decomposition we shall call a selectible arc of pseudo-arcs (a selectible circle of pseudo-arcs), or shortly, an s-arc of pseudo-arcs and an s-circle of pseudo-arcs. Thus we have obtained the following theorem. 
Theorem 8. There exist a plane s-arc of pseudo-arcs and a plane s-circle of pseudo-arcs.

In 8 Lewis asked whether curves admitting continuous decomposition into pseudo-arcs with an arc as the quotient space are topologically unique (Question 1 ), and more specifically, whether the elements of such a decomposition must be terminal (Question 2). The above examples show that the answer to both these question is no. The known arcs of pseudo-arcs (circles of pseudo-arcs) are chainable (circularly chainable), while the examples constructed here are triodic, and thus topologically different. Evidently, their elements of the decompositions are not terminal.

One can verify that the constructed s-arcs of pseudo-arcs do not separate the plane, and thus they are tree-like. We observe that this is a property of each s-arc of pseudo-arcs.

Proposition 9. Each s-arc of pseudo-arcs is a tree-like continuum.

Indeed, this is a consequence of a theorem of Sher on upper semi-continuous decompositions of compacta with elements of trivial shape ([13], Theorem 11).

Corollary 10. Each s-arc of pseudo-arcs has only one selector.

In view of the above conclusions the following question seems to be interesting.

Question 1. Are all s-arcs of pseudo-arcs topologically equivalent?

It is easy to see that for each graph $G$ we can construct a curve $\tilde{G}$ with continuous decomposition into pseudo-arcs having a continuous selector homeomorphic to $G$. Indeed, we represent $G$ as the union of a finite family of $\operatorname{arcs} p_{i} q_{i}$ that intersect only at end-points. Then we take a corresponding family of mutually disjoint s-arcs of pseudo-arcs with selectors $p_{i}^{\prime} q_{i}^{\prime}$, identify corresponding end-points of the selectors, and extend this identification to all the end elements of the decompositions. Now, following the idea of Lewis from [7], we can prove the next theorem.

Theorem 11. For each curve $M$ there exists a curve $\tilde{M}$ having a continuous decomposition into pseudo-arcs with a continuous selector $M^{\prime}$ of this decomposition, homeomorphic to $M$.

Proof. We represent $M$ as $\lim \left(G_{n}, f_{n}\right)$, where each $G_{n}$ is a graph and each $f_{n}$ is a piecewise linear, finite-to-one mapping. Then we inductively construct continuous curves $\tilde{G}_{n}$ of pseudo-arcs having $G_{n}$ 's as the continuous selectors with the natural mapping $q_{n}: \tilde{G}_{n} \rightarrow G_{n} \subset \tilde{G}_{n}$ being open and monotone retractions, and extensions $\tilde{f}_{n}: \tilde{G}_{n} \rightarrow \tilde{G}_{n-1}$ so that the following diagram commutes:

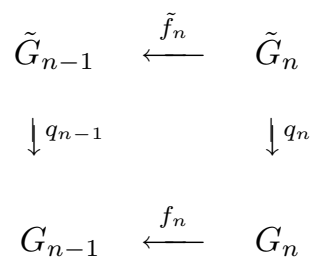

Indeed, we construct $\tilde{G}_{1}$ and the retraction $q_{1}: \tilde{G}_{1} \rightarrow G_{1}$ as described just before the formulation of the theorem. 
Assume that for some $n \geq 1$ the space $\tilde{G}_{n}$ and the mapping $q_{n}: \tilde{G}_{n} \rightarrow G_{n}$ is defined. Consider $f_{n+1}: G_{n+1} \rightarrow G_{n}$, where $G_{n+1}$ and $G_{n}$ are the unions of finitely many free arcs

$$
\begin{gathered}
G_{n}=a_{1} b_{1} \cup \ldots \cup a_{k} b_{k}, \\
G_{n+1}=c_{1} d_{1} \cup \ldots \cup c_{m} d_{m},
\end{gathered}
$$

such that each pair of $\operatorname{arcs} a_{\alpha} b_{\alpha}, a_{\beta} b_{\beta}$ and $c_{\gamma} d_{\gamma}, c_{\delta} d_{\delta}$ has at most one end point in common (for $\alpha \neq \beta$ and $\gamma \neq \delta$ ), and there is a function

$$
\phi:\{1, \ldots, m\} \rightarrow\{1, \ldots, k\}
$$

such that the mapping

$$
f_{n+1} \mid c_{\gamma} d_{\gamma}: c_{\gamma} d_{\gamma} \rightarrow a_{\phi(\gamma)} b_{\phi(\gamma)}
$$

is a homeomorphism. To each arc $a_{\alpha} b_{\alpha} \subset G_{n}$ we define the s-arc of pseudo$\operatorname{arcs} P_{\alpha}=q_{n}^{-1}\left(a_{\alpha} b_{\alpha}\right)$. Further, to each $\operatorname{arc} c_{\gamma} d_{\gamma} \subset G_{n+1}$ in the union (2), we attach an s-arc of pseudo-arcs $Q_{\gamma}$ such that $c_{\gamma} d_{\gamma} \subset Q_{\gamma}$ is the selector of $Q_{\gamma}$ and $Q_{\gamma} \cap Q_{\delta}=c_{\gamma} d_{\gamma} \cap c_{\delta} d_{\delta}$ for $\gamma \neq \delta$ in such a manner that there is a homeomorphism $h_{\gamma}: Q_{\gamma} \rightarrow P_{\phi(\gamma)}$ satisfying $h_{\gamma}\left|c_{\gamma} d_{\gamma}=f_{n+1}\right| c_{\gamma} d_{\gamma}$. Let $Y=\bigcup\left\{Q_{\gamma}: \gamma \in\{1, \ldots, m\}\right\}$.

Define two auxiliary mappings on $Y$ : the first, $g_{n+1}: Y \rightarrow G_{n+1}$, is the natural projection (being a monotone retraction), and the second, $f_{n+1}^{\prime}: Y \rightarrow \tilde{G}_{n}=$ $\bigcup\left\{P_{\alpha}: \alpha \in\{1, \ldots, k\}\right\}$, is defined by

$$
f_{n+1}^{\prime}(y)=h_{\gamma}(y) \text { for } y \in Q_{\gamma} .
$$

Consider an equivalence relation $\star$ on $Y$ for each $y_{1}, y_{2} \in Y$, putting $y_{1} \star y_{2}$ provided that

$$
g_{n+1}\left(y_{1}\right)=g_{n+1}\left(y_{2}\right) \text { and } f_{n+1}^{\prime}\left(y_{1}\right)=f_{n+1}^{\prime}\left(y_{2}\right) .
$$

Then the quotient space $Y / \star$ is just $\tilde{G}_{n+1}$, and the mappings $\tilde{f}_{n+1}: \tilde{G}_{n+1} \rightarrow \tilde{G}_{n}$ and $q_{n+1}: \tilde{G}_{n+1} \rightarrow G_{n+1}$ are defined in a natural way so that the diagram

$$
\begin{aligned}
& \text { Y } \\
& f_{n+1}^{\prime} \swarrow \quad \searrow^{g_{n+1}} \\
& \tilde{G}_{n} \stackrel{\tilde{f}_{n+1}}{\longleftarrow} \tilde{G}_{n+1}=Y / \star \stackrel{q_{n+1}}{\longrightarrow} G_{n+1} \\
& q_{n} \searrow \quad \swarrow f_{n+1} \\
& G_{n}
\end{aligned}
$$

commutes. The reader can verify in a routine way that $\tilde{G}_{n+1}$ and $\tilde{f}_{n+1}$ have the required properties.

We define $\tilde{M}=\lim _{\leftarrow}\left(\tilde{G}_{n}, \tilde{f}_{n}\right)$ and we observe that $M=\lim _{\leftarrow}\left(G_{n}, f_{n}\right)$ is contained in $\tilde{M}$. Since each $q_{n}: \tilde{G}_{n} \rightarrow G_{n}$ is a monotone, open retraction, so is the mapping $q=\lim _{\leftarrow} q_{n}: \tilde{M} \rightarrow M$. Denote by $\pi_{n}: \tilde{M} \rightarrow \tilde{G}_{n}$ the projection choosing the $n$-th coordinate. Note that for any $p \in M$ the sets $P_{n}(p)=q_{n}^{-1}\left(\pi_{n}(p)\right)$ are pseudo-arcs, while the mappings $\tilde{f}_{n} \mid P_{n+1}(p): P_{n+1}(p) \rightarrow P_{n}(p)$ are homeomorphisms. This implies that the set $q^{-1}(p)=\lim _{\leftarrow}\left(P_{n}(p), \tilde{f}_{n} \mid P_{n+1}(p)\right)$ is a pseudo-arc. The proof is complete. 
A curve $C$ will be called an s-curve of pseudo-arcs (a selectible curve of pseudoarcs), provided $C$ admits a continuous decomposition into pseudo-arcs, and $C$ contains a continuous selector of this decomposition. Thus the continua $\tilde{M}$ guaranteed by Theorem 11 are s-curves of pseudo-arcs. Similarly as in Proposition 9 we obtain the following.

Proposition 12. If $X$ is an s-curve of pseudo-arcs with a continuous selector $S$, then $X$ is a tree-like continuum if and only if $S$ is tree-like.

Proposition 13. If $X$ is a tree-like s-curve of pseudo-arcs, then its continuous selector is uniquely determined. Moreover, for such $X$, its continuous decomposition into pseudo-arcs admitting a continuous selection is unique.

Proof. Suppose $S_{1}$ and $S_{2}$ are continuous selectors of a tree-like s-curve of pseudo$\operatorname{arcs} X$ with some point $x \in S_{1}-S_{2}$. Take a continuum $K \subset S_{1}-S_{2}$ containing $x$ and some point $y \neq x$. Then there are pseudo-arcs $P_{x}, P_{y}$ containing $x$ and $y$, respectively, such that $\left(P_{x}-\{x\}\right) \cup\left(P_{y}-\{y\}\right) \subset\left(X-S_{1}\right)-S_{2}$. Thus the continuum $L=P_{x} \cup K \cup P_{y} \subset X-S_{2}$ is decomposable. Therefore it must intersect two different pseudo-arcs $P_{1}, P_{2}$ from the decomposition associated with the selector $S_{2}$. Observe that the continuum $L \cup P_{1} \cup S_{2} \cup P_{2} \subset X$ is not unicoherent, an impossibility.

Let $S$ be the unique continuous selector of $X$. Suppose there are two different continuous decompositions $\mathcal{D}_{1}$ and $\mathcal{D}_{2}$ of $X$ into pseudo-arcs such that $S$ is the continuous selector of each of them. Then there are a point $p$ in $X-S$, two different points $x$ and $y$ in $S$, and two pseudo-arcs $P_{1} \in \mathcal{D}_{1}$ and $P_{2} \in \mathcal{D}_{2}$ such that $x \in P_{1}, y \in P_{2}$ and $p \in P_{1} \cap P_{2}$. This implies that $\left(P_{1} \cup P_{2}\right) \cap S=\{x, y\}$, and consequently the union $S \cup P_{1} \cup P_{2} \subset X$ is not unicoherent, an impossibility by the tree-likeness of $X$. The proof is complete.

The following question naturally arises.

Question 2. Must an arbitrary s-curve of pseudo-arcs have only one continuous selector?

Now we investigate the topological properties of the selectors of plane s-curves of pseudo-arcs. Suppose an arc $p q$ is contained in the continuous selector $S$ of a plane s-curve of pseudo-arcs $X$ with the quotient mapping $g: X \rightarrow S$. Let $\left\{x_{n}\right\}$ be a sequence in $p q$ converging to some $x_{0} \in p q-\{p, q\}$. Take an open ball $N_{\varepsilon}\left(x_{0}\right)$ such that $p, q \notin N_{\varepsilon}\left(x_{0}\right)$. Then the component $C$ of $p q \cap N_{\varepsilon}\left(x_{0}\right)$ containing $x_{0}$ separates $N_{\varepsilon}\left(x_{0}\right)$ into exactly two components, $U_{1}$ and $U_{2}$. Further, since $g^{-1}\left(x_{0}\right)$ is acyclic, all sufficiently small subcontinua of $g^{-1}\left(x_{0}\right)$ containing $x_{0}$ access $x_{0}$ from exactly one of these $U_{i}$ 's (say from $U_{1}$ ). Observe that for almost all $n$, all sufficiently small subcontinua of $g^{-1}\left(x_{n}\right)$ lie in $U_{1} \cup\left\{x_{n}\right\}$ by the continuity of the decomposition of $X$. Note that this implies the following proposition.

Proposition 14. No selector of any planar s-curve of pseudo-arcs contains a simple triod.

Now we prove the next proposition.

Proposition 15. Each selector of any planar s-curve of pseudo-arcs is hereditarily locally connected.

Indeed, let $X$ be a plane s-curve of pseudo-arcs with the selector $S$ and the quotient mapping $g: X \rightarrow S$. Suppose, on the contrary, that $S$ contains a nondegenerate continuum $K_{0}$ and subcontinua $K_{n}$ converging to $K_{0}$ with $K_{i} \cap K_{j}=\emptyset$ 
for all $i, j=0,1, \ldots$ Take three different points $a, b, c \in K_{0}$ and sequences $\left\{a_{n}\right\},\left\{b_{n}\right\},\left\{c_{n}\right\}$ converging to $a, b, c$, respectively, such that $a_{n}, b_{n}, c_{n} \in K_{n}$. Then the triods $K_{n} \cup g^{-1}\left(a_{n}\right) \cup g^{-1}\left(b_{n}\right) \cup g^{-1}\left(c_{n}\right)$ must intersect the triod $K_{0} \cup$ $g^{-1}\left(a_{0}\right) \cup g^{-1}\left(b_{0}\right) \cup g^{-1}\left(c_{0}\right)$ for almost all $n$, an impossibility.

Propositions 14 and 15 imply that the selectors of planar s-curves of pseudo-arcs are either arcs, or simple closed curves. We have obtained the following theorem.

Theorem 16. Each planar s-curve of pseudo-arcs is either an s-arc of pseudo-arcs, or an s-circle of pseudo-arcs.

As in the above construction of the plane s-arc of pseudo-arcs $g_{a}^{-1}(L)$ (taking different $a$ from $\left.\left(0, a_{0}\right]\right)$, for any s-arc of pseudo-arcs $X$ we obtain a continuous, monotone family $X_{t}$ of s-arcs of pseudo-arcs, $t \in(0, T]$, with $X=X_{T}$ and some $X_{0}$ as the common selector for all $X_{t}$, satisfying $X_{t} \subset X_{u}$ if and only if $t \leq u$.

Question 3. If $X_{1}, X_{2}$ are (planar) s-arcs of pseudo-arcs with the common continuous selector $X_{0}$ and with $X_{1} \subset X_{2}$, does it follow that there is a continuous mapping (a retraction) $f: X_{2} \rightarrow X_{1}$ with $f(x)=x$ for $x \in X_{0}$ ?

Finally, we ask two questions on self-homeomorphisms of s-arcs of pseudo-arcs. Assume that $X$ is an arbitrary (planar) s-arc of pseudo-arcs with the continuous selector $X_{0}$ and the quotient mapping $q: X \rightarrow X_{0}$.

Question 4. Given a homeomorphism $h: X_{0} \rightarrow X_{0}$, can $h$ be extended to a homeomorphism $h^{*}: X \rightarrow X$ ?

Question 5. Given a point $p \in X_{0}$ and a homeomorphism $h: q^{-1}(p) \rightarrow q^{-1}(p)$ with $h(p)=p$, can $h$ be extended to a homeomorphism $h^{*}: X \rightarrow X$ ?

Now we will present some further possible constructions that can be derived from Theorem 11, Namely, we construct some other curves with continuous decomposition into nondegenerate subcontinua having a continuous selector. In particular, we will see that the elements of such decompositions need not be indecomposable.

First, we take a pseudo-arc $P_{1}$ for the continuum $M$ in Theorem 11. Then we obtain a selectible pseudo-arc of pseudo-arcs $P_{2}=\tilde{M}$. Observe that $P_{2}$ is a decomposable, tree-like curve (Proposition 12) that is not embeddable into the plane (Theorem 16 and Proposition 13). Similarly, we apply Theorem 11 to $P_{2}$, obtaining thus a tree-like curve $P_{3}$ with $P_{2}$ as a continuous selector of its continuous decomposition into pseudo-arcs. Observe that $P_{3}$ admits also a continuous decomposition into continua each having properties similar to those of $P_{2}$ (we do not know if they are topologically unique), with $P_{1}$ as the quotient space. Continuing this procedure by transfinite induction (for limit ordinals we take inverse limits with the quotient mappings), for any countable ordinal $\alpha>0$ we obtain a metric, tree-like curve $P_{\alpha}$ such that $P_{\alpha+1}$ admits a continuous decomposition into pseudo-arcs with $P_{\alpha}$ as a continuous selector. We do not know whether the sequence $\left\{P_{\alpha}\right\}$ is topologically unique. However, one can prove that for a fixed such sequence, continua $P_{\alpha}$ and $P_{\beta}$ are nonhomeomorphic if $\alpha \neq \beta$.

Now we repeat the above construction taking any continuum $M_{0}$ with dimension $\leq 1$ instead of $P_{1}$, obtaining thus a transfinite sequence $\left\{M_{\alpha}\right\}$ for countable ordinals $\alpha \geq 0$. One can verify that for each pair of countable ordinals $\alpha \geq 0$ and $\beta>0$ the curve $M_{\alpha+\beta}$ admits a continuous decomposition into continua of type $P_{\beta}$ from the previous sequence, with $M_{\alpha}$ as the quotient space. 
I end the paper with the following two announcements. In a subsequent paper I will prove them, applying the constructions introduced in the present one.

Announcement 1. Each compact 2-manifold $M$ (with or without boundary) admits a continuous decomposition into pseudo-arcs with the quotient space homeomorphic to $M$.

Announcement 2. The Sierpiński universal plane curve $S$ admits a continuous decomposition into pseudo-arcs with the quotient space homeomorphic to $S$.

Added in proof. The proofs of the above announcements have already been published in the paper: J. R. Prajs, Continuous decompositions of Peano plane continua into pseudo-arcs, Fund. Math. 158 (1998), 23-40.

\section{REFERENCES}

[1] R.D. Anderson, On collections of pseudo-arcs, Abstract 337t, Bull. Amer. Math. Soc. $56(1950), 350$.

[2] R. D. Anderson and G. Choquet, A plane continuum no two of whose nondegenerate subcontinua are homeomorphic: an application of inverse limits, Proc. Amer. Math. Soc. 10(1959), 347-353. MR 21:3819

[3] R. H. Bing, Concerning hereditarily indecomposable continua, Pacific J. Math. 1(1951), 43-51. MR 13:265b

[4] R. H. Bing and F. B. Jones, Another homogeneous plane continuum, Trans. Amer. Math. Soc. 90(1959), 171-192. MR 20:7251

[5] M. Brown, Continuous collections of higher dimensional continua, Ph. D. Thesis, University of Wisconsin, 1958.

[6] B. Knaster, Un continu dont tout sous-continu est indecomposable, Fund. Math. 3(1922), 247-286.

[7] W. Lewis, Continuous curves of pseudo-arcs, Houston J. Math. 11(1985), 91-99. MR 86e:54038

[8] W. Lewis, The pseudo-arc, Contemporary Math. 117(1991), 103-123. MR 92h:54046

[9] W. Lewis and J. J. Walsh, A continuous decomposition of the plane into pseudo-arcs, Houston J. Math. 4(1978), 209-222. MR 58:2750

[10] List of the works achieved in the field of mathematics and sciences in Poland during the German occupation, 1939-1945, Polish Academy of Sciences and Letters, Kraków 1947. MR 10:276e

[11] E. E. Moise, An indecomposable plane continuum which is homeomorphic to each of its nondegenerate subcontinua, Trans. Amer. Math. Soc. 63(1948), 581-594. MR 10:56i

[12] R. L. Moore, Concerning upper semi-continuous collections of continua, Trans. Amer. Math. Soc. 27(1925), 416-428.

[13] R. B. Sher, Realizing cell-like maps in Euclidean space, General Topology and its Appl. 2(1972), 75-89. MR 46:2683

Institute of Mathematics, Opole University, ul. Oleska 48, 45-052 Opole, Poland

E-mail address: jrprajs@math.uni.opole.pl 